\title{
Accessing the ECG Data of the Apple Watch and Accomplishing Interoperability Through FHIR
}

\author{
Alexander BARTSCHKE ${ }^{\mathrm{a}, 1}$, Yannick BÖRNER ${ }^{\mathrm{b}}$ and Sylvia THUN ${ }^{\mathrm{a}, \mathrm{c}, \mathrm{d}}$ \\ ${ }^{\mathrm{a}}$ Charité - Universitätsmedizin Berlin, Germany \\ ${ }^{\mathrm{b}}$ Humboldt University of Berlin, Germany \\ ${ }^{\mathrm{c}}$ Berlin Institute of Health (BIH), Germany \\ ${ }^{\mathrm{d}}$ Hochschule Niederrhein - University of Applied Sciences, Krefeld, Germany
}

\begin{abstract}
Medical data generated by wearables and smartphones can add value to health care and medical research. This also applies to the ECG data that is created with Apple Watch 4 or later. However, Apple currently does not provide an efficient solution for accessing and sharing ECG raw data in a standardized data format. Our method aims to provide a solution that enables patients to share their Apple Watch's ECG data with any health care institution via an iPhone application. We achieved this by implementing a parser in Swift that converts the Apple Watch's raw ECG data into a FHIR observation. Furthermore, we added the capability of transmitting these observations to a specified server and equipping it with the patient's reference number. The result is a user-friendly iPhone application, enabling patients to share their Apple Watch's ECG data in a widely known health data standard with minimal effort. This allows the personnel involved in the patient's treatment to use data that was previously difficult to access for further analyses and processing. Our solution can facilitate research for new treatment methods, for example, utilizing the Apple Watch for continuous monitoring of heart activity and early detection of heart conditions.
\end{abstract}

Keywords. Interoperability, FHIR, Smart Watch, ECG data

\section{Introduction}

\subsection{Background}

Worldwide the use of smartwatches and smartphones to generate and collect healthrelated data is steadily increasing. The popularity of smartwatches has risen so enormously that Apple sold more smartwatches in the last quarter of 2016 than the entire Swiss watch industry, resulting in Apple dominating the entire watch market. Apple Watch Series 4 or later can record an electrocardiogram (ECG) and classify the cardiac rhythm as atrial fibrillation (AF) or sinus rhythm (SR). [1] Beside the detection of AF, ECG data can generally be used for other use cases. For example, artificial intelegence (AI) algorithms can use ECG data to make informed decisions on how to treat medical conditions [2,3], big data analyses can use the data to identify new clinical and biological coherences and electronic health record (EHR) Systems can improve monitoring of

\footnotetext{
${ }^{1}$ Corresponding Author, Alexander Bartschke, Charité - Universitätsmedizin Berlin, Anna-Louisa-Karsch-Strasse 2, 10178 Berlin, Germany; E-mail: alexander.bartschke@charite.de.
} 
patients. For these use cases however, the data has to be accessible and should be transferred between systems in a standard data format that can be handled by both humans and machines.

To achieve semantic interoperability six major technology companies - including Google - have adopted Fast Healthcare Interoperability Resources (FHIR) as the standard for exchanging healthcare information in 2018 [4]. FHIR is a standard for the exchange of health data developed by HL7. It defines about 140 general concepts in health care, known as resources. These are the basic components of FHIR and can be extended as needed. Resources can be retrieved and exchanged using modern web technology such as Representational State Transfer (REST) Application Programming Interface (API) [5].

With the arrival of iOS 8 every iPhone is preloaded with the Health app. This application serves as a hub for all health-related devices as well as applications and acts as a storage for all these related data. It also provides an easy-to-read, secure and accurate dashboard. Apple's developer framework HealthKit provides an API for access to the data stored in the Health app. HealthKit however does not facilitate access to raw ECG data. It only provides access to PDF files containing a visual representation of the ECGs' waveforms. The only method to access the raw ECG data is to export the user's entire health data via the health app. This poses a problem, as the data cannot be analyzed directly on the iPhone and is not provided in a standardized format. There are other projects that use FHIR for heart rate or other high frequency data communication. However, in these projects the transmission of raw ECG data is not included.[6]

\subsection{Requirements}

In order to enable the use cases described above the ECG data needs to be provided in a standard data format. Furthermore, the patient's involvement in sharing the data needs to be effortless, which is currently not possible given the constraints of HealthKit.

To address this lack of functionality we developed a method in the form of a userfriendly application which reads all the user's ECGs in one bulk operation, converts them into FHIR observations and subsequently sends these observations to a previously defined server. This entire process takes place on the user's iPhone and does not need additional equipment. To the authors' knowledge there are currently no other approaches to access ECG raw data of the Apple Watch directly via an iPhone.

\section{State of the art}

\subsection{ECG Recordings}

The Apple Watch Series 4 or later connects a titanium electrode in the Digital Crown and an ultra-thin chromium-silicon-carbon nitride layer applied to the back of the watch [7]. With the combination of these technical features and the ECG app - which is cleared by the Food and Drug Administration (FDA) as Class II medical device - a single lead ECG similar to Einthoven's lead I ECG can be recorded by taking the index finger of the hand contralateral to the wrist with the watch and placing the finger on the Digital Crown for 30 seconds [8]. The ECG app reads and records the electrical impulses that are created with every heart contraction. After recording, the user can also report symptoms.

Here they can choose one or more symptoms provided by the app, for instance dizziness, shortness of breath or fatigue. An algorithm classifies the ECG as AF, SR or 
inconclusive. This classification, average heart rate, the user-reported symptoms, and waveform are stored in HealthKit.

\subsection{Accessing the Data}

The ECG recordings can be obtained in Health app as a PDF file containing the waveform of the ECG. The PDF can be shared by the user from the app via a share button. However, such a PDF is not suitable for automatic data processing. Using the Health app also has the disadvantage that only one PDF can be selected/sent manually at a time, making data sharing inefficient.

The only method to access the raw ECG data is an export of the entire data of the Health app: Using the export function, Health app generates a zip archive containing all raw data of the app in XML format, including a folder containing comma-seperated value (CSV) files of all raw data of every ECG record. Each CSV contains 15350 individual measurement points and additional metadata shown in Table 1 (the zip archive does not contain the PDFs or any other visualization of the ECG records).

Table 1: Metadata of each ECG record

\begin{tabular}{|l|}
\hline Name \\
\hline Date of Birth \\
\hline Recorded Date \\
\hline Classification \\
\hline Symptoms \\
\hline Software Version \\
\hline Device \\
\hline Sample Rate \\
\hline Lead \\
\hline Unit \\
\hline
\end{tabular}

\section{Concept}

Our method for transmission of ECG data in the form of FHIR observations is based on accessing the export of the Health App through a so-called share extension. Share extensions are used within iOS in order to pass data between different applications. Through this share extension the CSVs are converted into FHIR observations and then sent to a server (Figure 1).

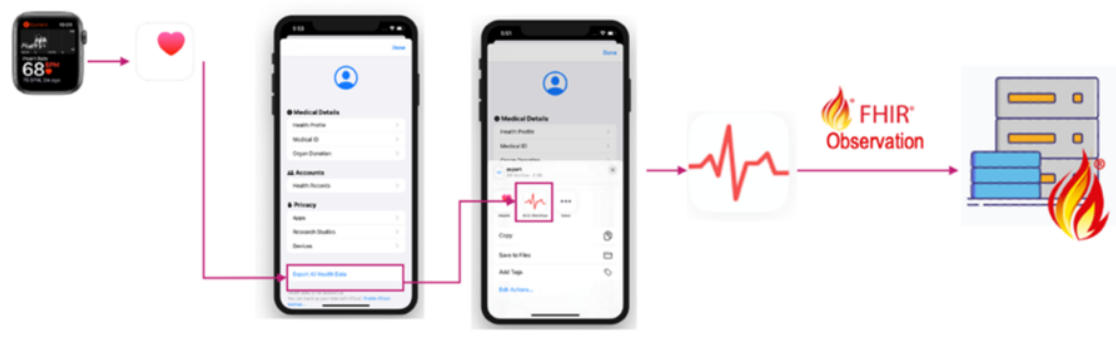

Figure 1: Data workflow 
Systematized Nomenclature of Medicine - Clinical Terms (SNOMED CT) are used to encode user-reported-symptoms shown in table 2. Logical Observation Identifiers Names and Codes (LOINC) are used to encode the metadata. In this context it must be mentioned that Germany does not have a SNOMED CT license yet. However, since March 2020 SNOMED CT can be used nationwide within the framework of the Medical Informatics Initiative (MII). In the near future, the Federal Ministry of Health plans to continue SNOMED CT in a current draft law, also for the care sector, in the form of a national license.[9]

Table 2: User-reported symptoms encoded with SNOMED CT

\begin{tabular}{|l|l|}
\hline Symptoms & SNOMED CT \\
\hline $\begin{array}{l}\text { Rapid pounding or } \\
\text { fluttering heartbeat }\end{array}$ & Palpitations (finding), SCTID: 80313002 \\
\hline Skipped heartbeat & Dropped beats - heart, SCTID: 248653008 \\
\hline Fatigue & Fatigue (finding), SCTID: 84229001 \\
\hline Shortness of breath & Dyspnea (finding), SCTID: 267036007 \\
\hline $\begin{array}{l}\text { Chest tightness } \\
\text { or pain }\end{array}$ & $\begin{array}{l}\text { Tight chest (finding) SCTID: } 23924001 \text { Chest } \\
\text { pain (finding) SCTID: 29857009 }\end{array}$ \\
\hline Fainting & Syncope (disorder), SCTID: 271594007 \\
\hline Dizziness & Dizziness (finding), SCTID: 404640003 \\
\hline Other & Other (qualifier value), SCTID: 74964007 \\
\hline None & None (qualifier value), SCTID: 260413007 \\
\hline
\end{tabular}

As specification of symptoms after ECG measurement is not enforced, it may be necessary to know the reason for missing information for later analysis of the data. In case no information regarding symptoms has been given, the observation notes that the user has been asked but declined to answer in accordance with the possible cases defined by FHIR extension Data Absent Reason. The submission of the referencing to the patient resource within the application ensures a correct assignment. Since the zip archive contains all data, it must be ensured that older measurements, which is every ECG that has been sent to the server already, are not sent to the server again after the second use of the application. This is ensured by assigning a unique ID to each ECG, which is persistently stored within the app, that can be marked as 'sent' or 'not sent'.

\section{Implementation}

The implementation at hand is written entirely in Swift and deployed via an iPhone application. It consists of two components, namely a main app and a share extension. The main app's sole purpose is capturing a server address and a patient reference as well as instructing the user how to use the app (Figure 2). The share extension is responsible for receiving the user's ECG data as well as the conversion to FHIR and subsequent transmission of the data to a server.

The main application is installed on the user's home screen like any other iPhone app. The user interface comprises two elements, namely two text fields for dynamically entering the server address and an instruction on how to transmit the data. The interface itself is scrollable, so that the application can be run on any iPhone with an arbitrary 
screen size. When exporting the health data from within the health app, the user is presented with an option to share the export with the application through our share extension.

When selecting the application, another screen is exposed, suggesting the user to transmit the data to the previously selected server.

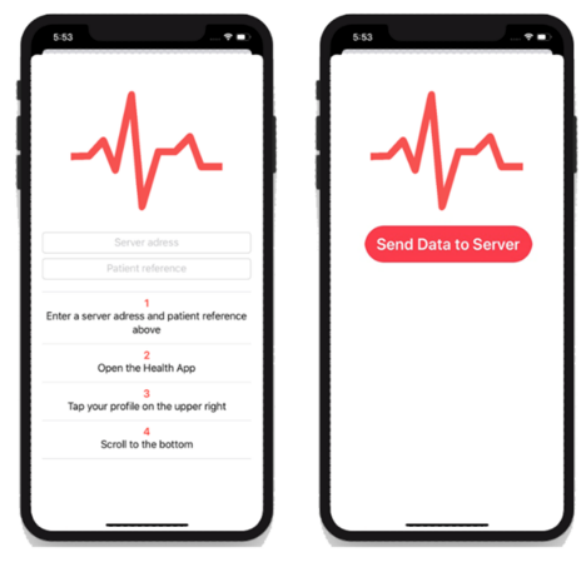

Figure 2: User interface

In case the user selects 'Send Data to Server' a few actions are triggered. (Figure 2) First, the entire export is extracted into a temporary directory within the applications main bundle. The application then selects every new ECG, and parses it via a series of steps. The first step is to read the ECG's corresponding CSV and extract all the metadata as well as the individual measurement points and symptoms. Subsequently, a FHIR resource is built via combining multiple JavaScript Object Notation (JSON) elements into a single FHIR observation. Utilizing the Sustainable Medical Applications, Reusable Technologies (SMART) on FHIR framework, the observation is then sent to the server address previously defined in the main application. Due to the fact that this application is a prototype, authorization, registration and privacy issues are not resolved yet. The code of the prototype is publicly available under https://github.com/ylboerner/FhirEcg.

\section{Lessons learned (Discussion)}

Our application meets the requirements described in 1.2 by enabling users to effortlessly share their ECG data with any entity within the health care system in a convenient way. Utilizing the FHIR standard ensures that the ECG data is ready for further analyses and processing. Our app thus improves the current way of sharing Apple Watch ECG data only through PDFs. After working with the FHIR standard, we can convincingly recommend it for transmitting ECG data as it will undoubtedly play a key role in future health care systems and is suitable for mobile applications with limited hardware resources. However, developing this solution came with certain challenges that needed to be addressed and required a great deal of communication with multiple parties. For instance, there was a bug in the export of the health data from within the Health app for users with their region set to Germany. This bug set the CSV's delimiter to a comma, 
which resulted in numbers with decimal places being interpreted as two numbers. Only after confirming this bug with Apple, we could advance with our implementation as the structure of the ECGs export was unclear before. Through this bug, we learned that technical challenges are still often solved through communication.

\section{Conclusion}

Our solution is a simple application, that allows users to share their ECG data with any entity, for instance a hospital or doctor's office, effortlessly. It adheres to the FHIR standard to ensure interoperability within any health care system, setting it up for future worldwide usage. Smartwatches with ECG functionality, like the Apple Watch, have the potential to considerably improve treatment of patients with cardiological conditions. However, these devices must have the ability to share their data with any healthcare provider in order to unlock their full potential. This is why interoperability should be of great interest to the manufacturers while designing their systems. During our research, we also learned that smartwatches are suitable for monitoring of heart activity. The accuracy of the Apple Watch is comparable to standard 12-lead ECG devices which opens up a range of new use cases for home monitoring and early detection of heart conditions.[9]

\section{Conflict of Interest}

The authors state that they have no conflict of interests.

\section{References}

[1] Massoomi MR, Handberg EM. Increasing and Evolving Role of Smart Devices in Modern Medicine, 14 (2019) 181-186. doi:10.15420/ecr.2019.02

[2] Cuocolo R, Perillo T, De Rosa E, Ugga L, Petretta M. Current applications of big data and machine learning in cardiology, 16(8) (2019) 601-607. doi:10.11909/j.issn.1671-5411.2019.08.002

[3] Mathews SM, Kambhamettu C, Barner KE. A novel application of deep learning for single-lead ECG classification,62 (2018) 99:53. doi:10.1016/j.compbiomed.2018.05.013

[4] Information Technology Industry Council, Cloud Healthcare Pledge (2018), https://www.itic.org/publicpolicy/CloudHealthcarePledge.pdf. (accessed April 5, 2020).

[5] D. Bender, K. Sartipi, HL7 FHIR: An Agile and RESTful Approach to Healthcare Information Exchange, Proceedings of the 26th IEEE International Symposium on Computer-Based Medical Systems (2013), $326-331$.

[6] Micrisoft/ iomt-fhir| (2019) https://github.com/microsoft/iomt-fhir (accessed July 18, 2020)

[7] Using Apple Watch for Arrhythmia Detection, (2018).https://www.apple.com/healthcare/docs/site/Apple_Watch_Arrhythmia_Detection.pdf. (accessed April 5, 2020).

[8] De Novo Classification Request for App , (2018). https://www.accessdata.fda.gov/cdrh_docs/reviews/DEN180044.pdf. (accessed April 5, 2020).

[9] SNOMED CT wird in Deutschland eingeführt, (2020). https://e-health-com.de/details-news/snomed-ct-wird-in-deutschland-eingefuehrt/ (accessed July 18, 2020)

[10] De Samol, A., Bischof K, Luani B, Pascut D, Wiemer M, Kaese S. Recording of Bipolar Multichannel ECGs by a Smartwatch: Modern ECG Diagnostic 100 Years after Einthoven. Sensors (Basel). 2019;19(13):2894. Published 2019 Jun 30. doi:10.3390/s19132894 